Rapid Reviews COVID-19

\title{
Review 1: "Scent dog identification of SARS- CoV-2 infections, similar across different body fluids"
}

\section{Albertini Mariangela ${ }^{\mathbf{1}}$, Patrizia Piotti $^{\mathbf{2}}$}

${ }^{1}$ Associated Professor, University of Milan: Universita degli Studi di Milano, Department of Veterinary Medicine, Italy,

${ }^{2}$ Department of Veterinary Medicine, University of Milan, Italy

Published on: Mar 31, 2021

License: Creative Commons Attribution 4.0 International License (CC-BY 4.0). 


\section{$\underline{\text { RR:C19 Evidence Scale rating by reviewer: }}$}

- Potentially informative. The main claims made are not strongly justified by the methods and data, but may yield some insight. The results and conclusions of the study may resemble those from the hypothetical ideal study, but there is substantial room for doubt. Decision-makers should consider this evidence only with a thorough understanding of its weaknesses, alongside other evidence and theory. Decisionmakers should not consider this actionable, unless the weaknesses are clearly understood and there is other theory and evidence to further support it.

$* * * * * * * * * * * * * * * * * * * * * * * * * * * * * * * * * * * * * * *$

\section{Review:}

This paper analyzes the ability of dogs to perceive the presence of VOCs linked to SARS-CoV-2 infection in different sample types, such as saliva, urine, and sweat taken from infected people.

It has already been widely proven that dogs are very skilled in perceiving the presence of VOCs linked to diseases of different types, not only infectious diseases but also, for example, different kinds of tumors. It is known that dogs can be considered a rapid, non-invasive, and relatively inexpensive screening tool that allows the detection of the presence of pathologies in a short time and, very importantly, dogs can work in very different and crowded places, such as airports, schools, etc.

It would therefore be of extreme importance to be able to train dogs to detect the presence of VOCs linked to the SARS-CoV-2 infection, and I believe this is absolutely feasible. However, I have several concerns about the methodology and consequently the results presented in this paper.

First of all, the experimental design is not well-described. The authors say they took samples from 93 subjects. Looking at table 1, it appears that these include 46 positive subjects and 47 negative subjects. The authors write that all of the samples used in the final study had not been presented to the dogs before and that, in each trial, the device 's software randomly assigned the target scent's position to one of the seven positions available. This seems to suggest that the dog should find a positive sample among the seven samples present, in a 1:6 ratio between positive and negative samples. However, when looking at the tables where the responses of the dogs are reported, it appears that each dog is presented with 19 to 21 positive samples, while the number of 
negative samples varies between 58 and 112. Since there were 47 negative subjects, does this mean that dogs were presented with negative samples from the same subjects multiple times? It is not specified. It is also not clear why some dogs were presented with 21 positive versus 58 negative samples, while other dogs were presented with 21 positive versus 112 negative samples. What is the reason for such a huge discrepancy in the number of negative samples? And, since the odds of indicating the correct sample decrease with the increase of the positive-negative ratio (thus making the task much more difficult), this discrepancy makes it very difficult to compare the abilities of each dog.

Another issue that I believe cannot be overlooked, is the behavioral criterion adopted by the authors to identify the dogs' response. I am very skeptical that seeing the dog sniffing the location for more than 2 seconds is sufficient to conclude that they recognized the positive sample. I do not recommend this should be considered sufficient as signaling. I believe it would be safer to teach the dog to signal more clearly, perhaps by sitting in front of the sample deemed positive. Furthermore, there are important gaps in the procedure as it is described. Were the dogs required to smell all samples before signaling the positive one? Or could they avoid smelling the other samples once they found the positive one? If that is the case, how can it be said that the dogs recognize negative samples as negative when they have not even smelled them? If the dogs did not smell all the presented samples, as a matter of fact, the Sp and Se cannot be calculated on the basis of the presented samples but only the examined ones. Additionally, the discrimination abilities would be greatly affected by the location of the positive sample (first versus last smelled).

I strongly agree with the authors' conclusions that detection dogs can provide a reliable screening method for SARS-CoV-2 infections in various settings to generate immediate results. To fully evaluate the potential of the dog detection method, however, more accurate training is necessary. This is also necessitated by the fact that laboratory conditions are very different from real-life conditions. And, above all, working on samples is very different than working directly on human beings, especially in environments where many individuals have to be screened.

Please find below some detailed comments:

- The title should be changed to "Scent dog identification of SARS-CoV-2 infections is similar across different body fluids." 
- The authors often refer to "canines" when they should rather refer to "dogs" or "domestic dogs," as they do not use other types of canines.

- In the methods section, it is not clear which samples are not inactivated.

- In the training section, there were 12 positive inactivated samples but it is not clear how many negative samples there are. 12 ? Please specify.

- What is the final study exactly? Please describe the protocol more clearly, describing and naming each phase.

- The transfer from inactivated to non-inactivated saliva samples is not clear, were they still using the DDTS? How was the trial performed and assessed?

- How many double-blind sessions the dogs underwent in total? How many for each substrate?

- L190: Please do not start sentences with a number (line 190)

- L197: The dogs were able...

- L201: Do the authors mean with different body fluids?

- L207-210: Please correctly refer to the results as FP, FN, etc.

- L210-212: Does that mean that the majority of positive samples were positive only for the nasopharyngeal swabs? Or what? Please specify. 